Check for updates

Cite this: RSC Adv., 2018, 8, 5312

Received 20th December 2017 Accepted 24th January 2018

DOI: 10.1039/c7ra13516e

rsc.li/rsc-advances

\section{Highly transparent and flexible polyaniline mesh sensor for chemiresistive sensing of ammonia gas $\uparrow$}

\begin{abstract}
Jingxuan Cai, ${ }^{\text {ab }}$ Cuiping Zhang, ${ }^{\text {ab }}$ Arshad Khan, ${ }^{a}$ Chuwei Liang ${ }^{\text {ab }}$ and Wen-Di Li (D) *ab
A new structure of a flexible, transparent polyaniline (PANI) ammonia gas sensor is reported. The sensor features a hierarchical nanostructured PANI polymer arranged in a micromesh, exhibiting excellent chemiresistive sensitivity to ammonia gas and near-neutral color transparency. The PANI mesh is embedded in a flexible substrate and therefore exhibits superior mechanical stability against peeling and bending. These merits make it a promising candidate for application in wearable electronics. Moreover, the PANI mesh sensor is fabricated through a cost-effective, solution-based strategy that enables vacuum-free fabrication of a sacrificial catalytic copper mesh followed by in situ polymerization, and this strategy is scalable for high-volume production. We demonstrate the high-performance resistive sensing of ammonia gas with concentrations from $2.5 \mathrm{ppb}$ to $100 \mathrm{ppm}$ using this flexible PANI mesh sensor with an excellent transparency of $88.4 \%$ at $600 \mathrm{~nm}$ wavelength. Furthermore, no significant degradation in the sensing performance occurs after 1000 bending cycles.
\end{abstract}

\section{Introduction}

Recently, due to the increasing demand for portable health monitoring applications, wearable electronic devices, such as electronic noses, ${ }^{1}$ smart skin, ${ }^{2}$ and smart wristbands, ${ }^{3}$ have been extensively studied. High-performance compact bio- and gas sensors are key for these applications, and efforts have been devoted to developing sensors meeting these demands. For example, a nanoplasmonic immunosensor has been constructed on few-layers $\mathrm{MoS}_{2}$ for cytokine optoelectronic immunosensing. ${ }^{4}$ Graphene nanoribbon arrays for $\mathrm{NO}_{2}$ gas sensing, ${ }^{5}$ silver nanowires-embedded polydimethylsiloxane electrode for pressure sensing, ${ }^{6}$ silicon multi-nanochannel FETs for detection of insulin in serum, ${ }^{7}$ and fluorescence resonance energy transfer based sensors utilizing conjugated polyelectrolyte for DNA sensing, ${ }^{8}$ have also been investigated. To fulfill the requirements of portable and real-time detection of trace amounts of hazardous gases in wearable health monitoring devices, high-performance thin-film sensors with excellent flexibility and transparency to be integrated in wearable optoelectronic devices are desirable. With a transparent appearance, the developed sensor can be better integrated into other devices with minimal interference of their optoelectronic functions. For example, ammonia is a volatile chemical that is widely used in various industries, and is a corrosive, poisonous and highly

${ }^{a}$ Department of Mechanical Engineering, The University of Hong Kong, Pokfulam, Hong Kong, China

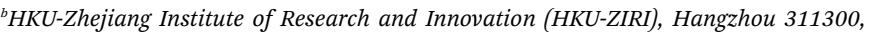
Zhejiang, China.E-mail: liwd@hku.hk

$\dagger$ Electronic supplementary information (ESI) available. See DOI: 10.1039/c7ra13516e toxic gas. Exposure to ammonia may cause severe harm to the skin, eyes or respiratory tract, and remaining in an environment with a high concentration of ammonia may even cause dyspnea or death. ${ }^{9}$ Thus, a flexible, highly sensitive sensor that is capable of detecting trace amounts of ammonia is desirable as an integrated wearable personal protection device during the safe handling of ammonia gas.

Most commonly used gas sensors are usually made from metal oxide semiconductors, which are often operated at elevated temperatures (above $200{ }^{\circ} \mathrm{C}$ ) and lack flexibility or transparency. ${ }^{10,11}$ The development of different gas sensors based on nanostructured materials for the efficient and rapid detection of trace harmful gases has recently become a fastgrowing field. In particular, electrically conductive nanostructured polymers have been suggested as promising candidates for chemiresistive gas sensors due to the strong response of their conductivity to specific gases. ${ }^{12-14}$ Among the conductive polymers, polyaniline (PANI), which is operated at room temperature and is $\mathrm{pH}$-sensitive, has been extensively studied because of its simple synthesis, high sensitivity, good flexibility and excellent reliability. ${ }^{15}$ Fabrication of flexible, transparent ammonia gas sensors based on PANI has recently been reported. For example, hierarchical nanostructured PANI networks have been fabricated on a polyethylene terephthalate (PET) film using a sacrificial silver nanowire template. ${ }^{16}$ The hierarchical nanostructured PANI networks exhibited 65\% transmittance at $550 \mathrm{~nm}$ and a sensitivity of 9 , which is defined as the relative change in the electrical resistance of ammonia gas sensors, under exposure to ammonia gas of $10 \mathrm{ppm}$. Hybrid structures combining PANI with graphene, ${ }^{17}$ carbon nanotubes, ${ }^{18}$ and reduced graphene oxide ${ }^{19}$ as well as inorganic 
materials such as zinc oxide $\mathrm{e}^{20}$ and titanium oxide ${ }^{21}$ have also been investigated. However, in many existing works, the procedure for fabricating the sensor is still complicated and expensive, the optical transmittance of the sensors is not satisfactory, and the reproducibility remains a challenge. Currently, most PANI sensors are constructed through catalystfree routes that produce PANI with low yields. A limited number of reports are available on the use of inorganic $\mathrm{Cu}(\mathrm{II}) \mathrm{salts}^{22}$ and a cis-bis(glycinato)copper(II) complex ${ }^{23}$ for the synthesis of PANI. However, the random distribution of the catalyst often leads to overoxidation to the pernigraniline form of PANI and the generation of various other by-products. These limitations call for improved and scalable fabrication process and new device structures for gas sensors that are desirable for wearable environmental monitoring devices.

Herein, we report the templated fabrication of a transparent chemiresistive sensing film of a hierarchical nanostructured PANI micromesh via in situ polymerization of aniline on a sacrificial $\mathrm{Cu}$ micromesh template embedded in a cyclic olefin copolymer (COC) substrate. The template $\mathrm{Cu}$ micromesh on COC is fabricated by photolithography, electrodeposition and subsequent thermal imprint transfer. The catalytic $\mathrm{Cu}$ micromesh not only provides a guided template for the selective deposition of nanostructured PANI but also enhances the deposition yield of the PANI, thus enhancing the transparency of the gas sensor without sacrificing its sensing performance. The assembled sensor exhibits high-performance sensing of ammonia gas with concentrations ranging from $2.5 \mathrm{ppb}$ to $100 \mathrm{ppm}$ and excellent near-neutral color transparency $(88.4 \%$ at $600 \mathrm{~nm}$ ). The embedded structure also improves the mechanical stability of the sensor under peeling and high bending stress, with no significant decrease in the sensing performance observed after 1000 bending cycles.

\section{Experimental}

\section{Material}

Aniline was purchased from J\&K Chemical (Hong Kong, China) and used without further purification. Ammonium peroxodisulfate (APS) and perfluorodecyltrichlorosilane (FDTS) were obtained from Alfa Aesar (Lancashire, United Kingdom). Hydrochloric acid was obtained from VWR (Batavia, USA). The COC film (Grade 8007) was commercially available from TOPAS Advanced Polymer (Frankfurt, Germany). Indium tin oxide (ITO) glass was obtained from South China Xiang Science \& Technology (Shenzhen, China). Cu sulfate pentahydrate, sulfuric acid, and sodium dodecyl sulfate were purchased from Acros Organics (Geel, Belgium). Ethanol, 2-propanol, acetone, acetic acid, formaldehyde, toluene, and sodium bicarbonate were purchased from J\&K Chemical (Hong Kong, China).

\section{Preparation of $\mathrm{Cu}$ mesh}

The preparation method for the $\mathrm{Cu}$ mesh was modified from our previously published method. A hexagonal pattern was first fabricated on a $1.8 \mu \mathrm{m}$-thick AZ1500 photoresist-coated ITO glass substrate $\left(3 \times 3 \mathrm{~cm}^{2}\right)$ after exposure to a $365 \mathrm{~nm}$ UV source $\left(55.5 \mathrm{~mJ} \mathrm{~cm}^{-2}\right)$ through a photomask. A $1.5 \mu \mathrm{m}$-thick hexagonal $\mathrm{Cu}$ mesh was then electrodeposited in the trench of the photoresist-coated ITO glass in a Cu electrolyte (Caswell, New York, USA) with a current density of $5 \mathrm{~mA} \mathrm{~cm}{ }^{-2}$. After removal of the photoresist with acetone, the $\mathrm{Cu}$ mesh was transferred to a COC film by hot embossing.

\section{Preparation of PANI mesh}

The $\mathrm{Cu}$ mesh-embedded COC film was first treated by oxygen plasma for 30 seconds, and an FDTS self-assembled monolayer coating was then prepared on the film by a vapor phase deposition process operated at $95{ }^{\circ} \mathrm{C}$ for $15 \mathrm{~min}$. The PANI mesh on the COC film was fabricated via in situ chemical oxidative polymerization of aniline. In a typical procedure, the aniline monomer $(2.4 \mathrm{mmol})$ was added into a precooled $1 \mathrm{M}$ hydrochloric acid aqueous solution $(20 \mathrm{~mL})$ in a beaker and dispersed using ultrasonic vibration at $0.5{ }^{\circ} \mathrm{C}$. Then, $20 \mathrm{~mL}$ precooled aqueous solution of $1 \mathrm{M}$ hydrochloric acid and $1 \mathrm{M}$ APS was poured into the aniline monomer solution, and the mixture was shaken for 30 seconds. Afterward, the $\mathrm{Cu}$ mesh-embedded COC film was immersed in the solution for the etching of $\mathrm{Cu}$ and the polymerization and deposition of aniline with the existence of oxidizing APS. The temperature of the polymerization process was maintained at approximately $0.5^{\circ} \mathrm{C}$ by an ice bath. After immersion for various lengths of time, the polymerized COC films were thoroughly rinsed with DI water and ethanol, dried under a flow of nitrogen and baked in an oven at $70{ }^{\circ} \mathrm{C}$ for 30 min to remove absorbed water.

\section{Gas sensing measurements}

The gas sensing measurements were performed on a WS-30A sensing system (Zhengzhou Winsen Electronics Technology, China) equipped with an $18 \mathrm{~L}$ chamber, a hot plate and two fans for mixing the gas. The relative humidity and temperature were maintained at approximately $40 \%$ and $24{ }^{\circ} \mathrm{C}$ during the experiments. The PANI mesh-embedded COC films were cut to $1.5 \times$ $1.5 \mathrm{~cm}^{2}$ and attached to probes installed in the sensing system using silver paste. Calculated amounts of ammonium hydroxide (25 wt $\% \mathrm{NH}_{3}$ in $\mathrm{H}_{2} \mathrm{O}$ ) were dropped onto the hot plate in the chamber by a pipette to rapidly generate $5 \mathrm{ppm}, 10 \mathrm{ppm}$, $50 \mathrm{ppm}$, and $100 \mathrm{ppm}$ ammonia gas in the chamber, and the resistance of the film during exposure to a certain concentration of ammonia gas was recorded by the sensing system. To generate $100 \mathrm{ppb}, 200 \mathrm{ppb}, 500 \mathrm{ppb}$, and $1000 \mathrm{ppb}$ ammonia gas, $1 \mathrm{wt} \%$ ammonia hydroxide was used. To generate $2.5 \mathrm{ppb}, 5$ ppb, $10 \mathrm{ppb}$, and $25 \mathrm{ppb}$ ammonia gas, $0.1 \mathrm{wt} \%$ ammonia hydroxide was used. Thereafter, the PANI mesh was recovered under exposure to air by removing the chamber. To examine the gas sensing selectivity of the PANI mesh, other gas species, including ethanol, 2-propanol, acetic acid, acetone, formaldehyde, toluene, and carbon dioxide (generated from sodium bicarbonate) were tested by the same method.

\section{General characterization}

The morphology of the samples was characterized using an S4800N SEM (Hitachi, Japan) and an LEO 1530 Gemini SEM 
(Zeiss, Germany), and a Multimode-8 atomic force microscope (Bruker, USA). UV-vis transmittance spectra were collected on an HR2000+ ultraviolet/visible/near-infrared spectrometer (Ocean Optics, USA). All transmittance values presented in this paper were normalized to the absolute transmittance through the bare COC film substrate. Raman spectra were collected on a QE65 Pro Raman spectrometer (Ocean Optics, USA) using a $785 \mathrm{~nm}$ laser source. X-ray diffraction (XRD) spectra were recorded on a SmartLab X-ray diffractometer (Rigaku, Japan). The sheet resistance was measured by a Keithley 2400 source meter with a four-probe tester.

\section{Results and discussion}

Typically, COC films functionalized with a hierarchical PANI micromesh were fabricated through in situ polymerization of aniline on a $\mathrm{Cu}$ mesh-embedded COC film (Fig. 1). Fig. 1a displays a photo of a transparent PANI ammonia gas sensor prototype. The sensing mechanism of the PANI ammonia sensor is schematically illustrated in Fig. 1b. Upon exposure to a certain concentration of ammonia gas, ammonia molecules chemically bind to the protonated PANI, thus decreasing the conductivity of the PANI polymer by a reversible transition from the emeraldine salt form of PANI to its emeraldine base. ${ }^{24}$ Hence, by measuring the resistance across two silver electrodes on the transparent PANI gas sensor during exposure to ammonia gas, the concentration of the environmental ammonia gas can be determined.

The initial template $\mathrm{Cu}$ micromesh with a high transparency was fabricated on a $100 \mu \mathrm{m}$-thick COC (Grade 8007) film through a strategy consisting of photolithography, electrodeposition and an imprint transfer process, which has been thoroughly discussed in our previous report. ${ }^{25,26}$ The transparent electrode provides numerous merits, such as high conductivity and excellent flexibility, and a full-plastic bifacial dye-sensitized solar cell has been constructed on Ni-mesh electrode. ${ }^{27}$ The sheet resistance and transparency of the $\mathrm{Cu}$ mesh were $0.3 \Omega \mathrm{sq}^{-1}$ and $72 \%$, respectively (Fig. S1, ESI $\dagger$ ). The hexagonal $\mathrm{Cu}$ micromesh was embedded in the COC film and could be etched by ammonium persulfate (APS). Thus, the embedded $\mathrm{Cu}$ mesh could be employed as a sacrificial template for the in situ fabrication of flexible, highly transparent PANI mesh with excellent stability against mechanical deformation. Before the chemical polymerization process, the $\mathrm{Cu}$ mesh was treated with oxygen plasma for 30 seconds and coated with a self-assembled monolayer of perfluorodecyltrichlorosilane (FDTS) through vapor phase deposition to limit the deposition of PANI on the exposed surface of the COC film. Afterward, the $\mathrm{Cu}$ micromesh on COC was immersed in an aniline polymerization solution with an aniline concentration of $0.06 \mathrm{M}$ and a molar ratio of [aniline] : [APS] = $1.2: 1$ for various lengths of time (Fig. 1c(i)). The protonated hierarchical PANI mesh was then deposited onto the $\mathrm{Cu}$ sacrificial template (Fig. 1c(ii)). Finally, the PANI film that was deposited on the exposed COC film was removed with an adhesive tape (Fig. 1c(iii)) to complete the fabrication of the PANI mesh embedded in the COC film as an ammonia gas sensor (Fig. 1c(iv)).

Fig. 2 shows scanning electron microscopy (SEM) images of the Cu mesh (Fig. 2a and d) and the PANI mesh (Fig. 2b, c, e and f) at different polymerization stages. The hexagonal $\mathrm{Cu}$ micromesh was fully embedded in the COC film and showed a smooth structure, resulting in a high conductivity and excellent stability. The standard reduction potential of $\mathrm{Cu}(+0.34 \mathrm{~V})$ is lower than that of aniline $(+1.02 \mathrm{~V})$; thus, $\mathrm{Cu}$ is more likely than (a)

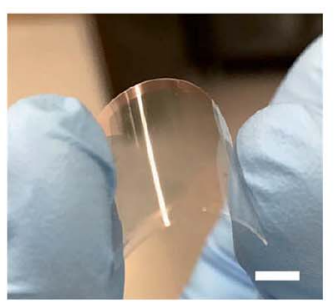

(b)

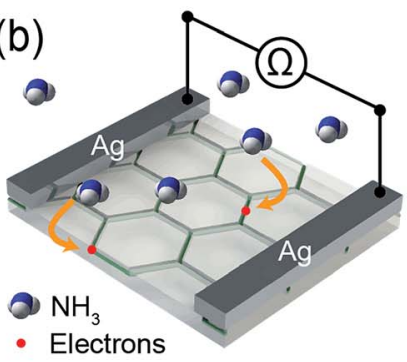

(c)
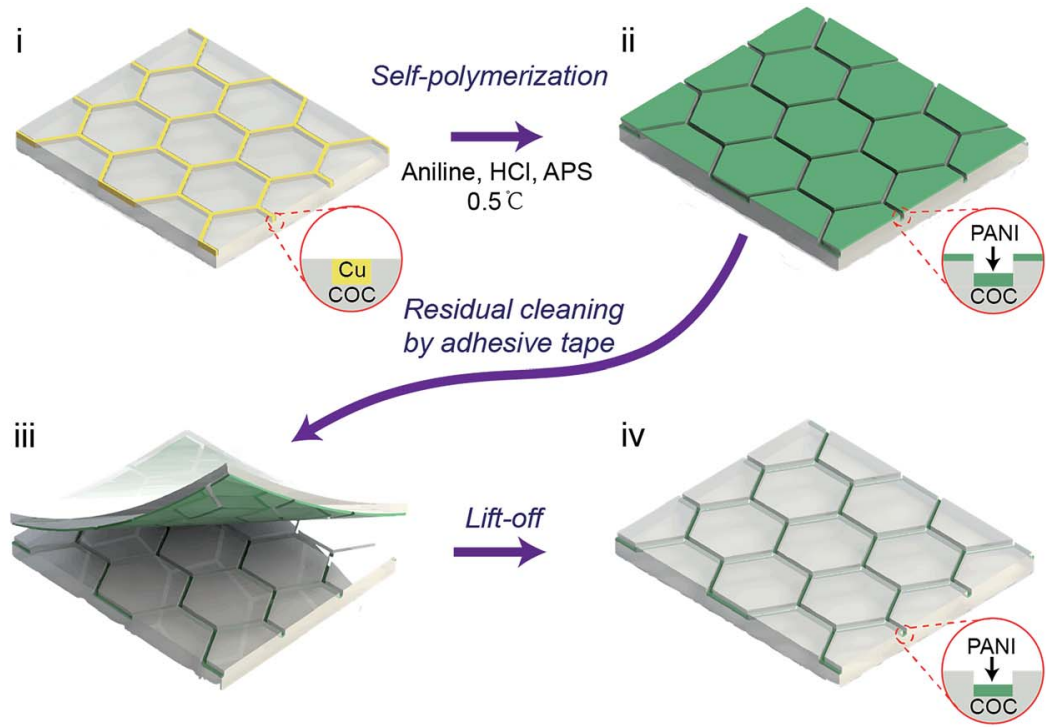

Fig. 1 Schematic illustration of the fabrication of a hexagonal hierarchical PANI micromesh via in situ polymerization of aniline on a sacrificial Cu micromesh template. (a) Photograph of the transparent PANI mesh ammonia gas sensor. (b) Sensing mechanism of the PANI ammonia sensor. (c) (i) The sacrificial Cu mesh template; (ii) deposition of the hierarchical PANI micromesh on the Cu template via in situ oxidative polymerization of aniline; (iii) removal of the excess residual PANI molecules from the exposed COC by adhesive tape; and (iv) a hexagonal hierarchical PANI micromesh embedded in the COC film. The scale bar represents $1 \mathrm{~cm}$. 

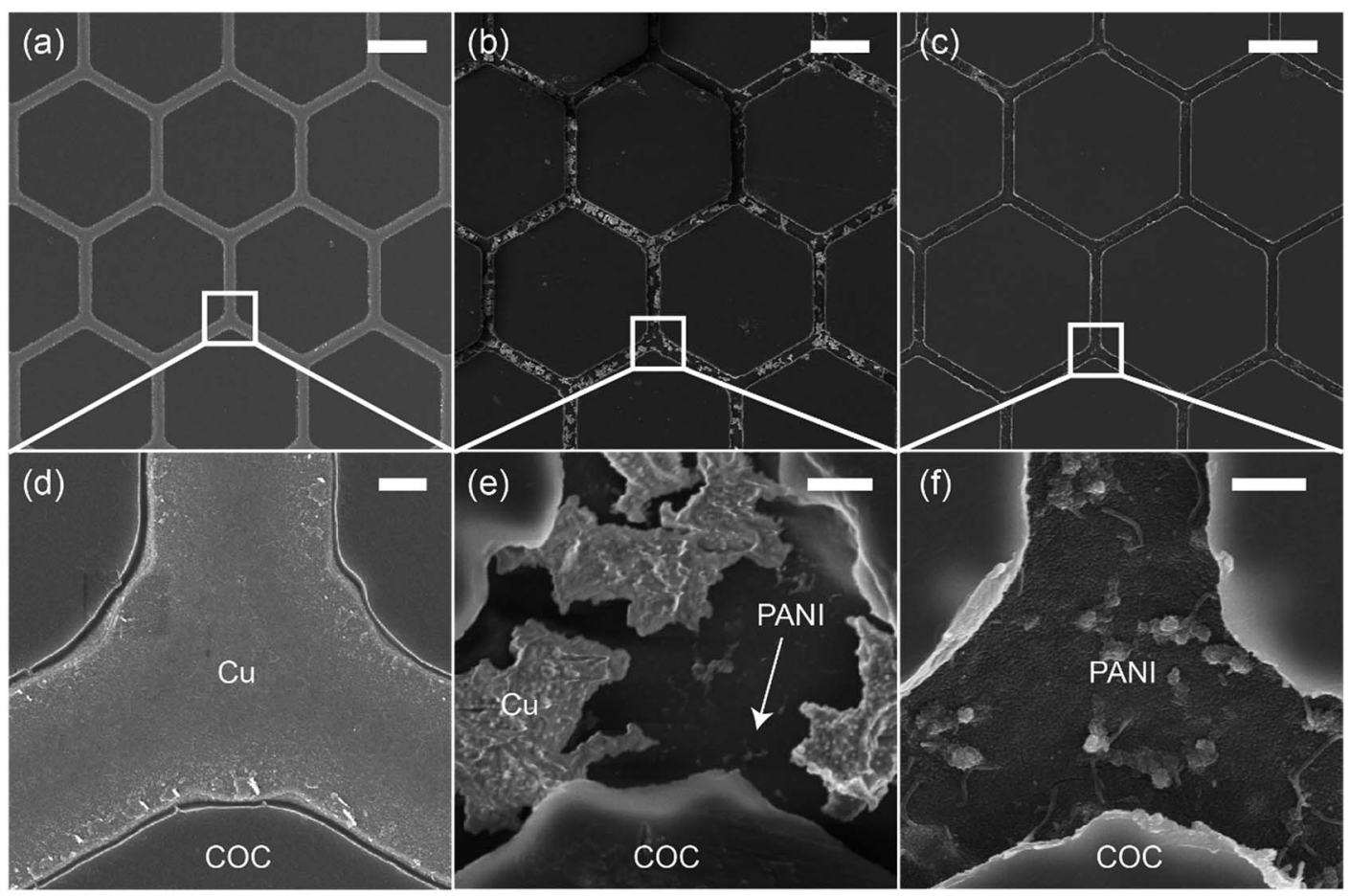

Fig. 2 SEM images of (a) the original hexagonal Cu mesh and the PANI mesh after (b) 3 min and (c) 20 min in the aniline polymerization solution, respectively. (d)-(f) Magnified images of the trench junction area in (a)-(c). The scale bars in (a)-(c) and (d) -(f) represent $20 \mu \mathrm{m}$ and $1 \mu \mathrm{m}$, respectively.

aniline to be oxidized by APS and forms $\mathrm{Cu}$ ions. As displayed in Fig. $2 \mathrm{~b}$ and $\mathrm{e}$, the $\mathrm{Cu}$ mesh was partially etched after immersion in the aniline polymerization solution for $3 \mathrm{~min}$. The etching process of $\mathrm{Cu}$ was also confirmed by energy-dispersive X-ray spectroscopy (EDS) analysis, $\mathrm{Cu}$ was etched away in the oxidizing APS solution and complex $\left[\mathrm{CuCl}_{4}\right]^{2-}$ was formed simultaneously (Fig. S2, ESI $\dagger$ ). For a longer immersion time of $20 \mathrm{~min}$, the $\mathrm{Cu}$ micromesh was completely etched away, and a hierarchical PANI micromesh consisting of PANI nanoparticles and nanofibers was deposited in the trenches containing the original sacrificial $\mathrm{Cu}$ template (Fig. 2c, f, and S3, ESI $\dagger$ ). EDS analysis revealed that trace of $\mathrm{Cu}$ was observed in the deposited PANI mesh, which confirmed the existence of oxidized $\mathrm{Cu}$ after the polymerization process (Fig. S4, ESI $\dagger$ ). A polymerization time of 20 min produced a PANI mesh with a thickness of approximately $100 \mathrm{~nm}$ (as measured by the atomic force microscope), and the average size of the deposited PANI nanoparticles was approximately $20 \mathrm{~nm}$ (Fig. S5, ESI $†$ ). Most of the PANI structures were deposited in the trenches rather than on the FDTS-treated COC surface because of the presence of catalytic $\mathrm{Cu}$ and $\mathrm{Cu}$ ions and the lower surface energy on COC surface. For comparison, an unmodified COC film was immersed in the aniline polymerization solution for 20 min, forming a PANI film with less particles and smoother surface, as shown in Fig. S6 (ESI $\dagger$ ).

The transparency and conductivity of the as-prepared PANI mesh changed with polymerization time because of the various amount of deposited PANI nanostructures. The optical transmittance of the PANI mesh after $10 \mathrm{~min}, 20 \mathrm{~min}$, and $30 \mathrm{~min}$ in the aniline polymerization solution are illustrated in Fig. 3a. The nanostructured PANI mesh showed an excellent optical transparency across the range of $500 \mathrm{~nm}$ to $900 \mathrm{~nm}$ with a maximum transmittance of $88.4 \%$, and this transparency was higher than that of the original $\mathrm{Cu}$ mesh $(72 \%)$ because of the semi-transparent nature of PANI. Moreover, as displayed in the insets of Fig. 3a, the assembled gas sensor exhibited a nearneutral color. For comparison, the PANI film after immersion in the aniline polymerization solution for $20 \mathrm{~min}$ exhibited a transmittance of only $56.3 \%$, and the film appeared green.

The sheet resistance of the PANI mesh changed with the time length of the oxidative polymerization and deposition of PANI on the COC substrate. As demonstrated in Fig. 3b, the sheet resistance of the original $\mathrm{Cu}$ mesh was $0.3 \Omega \mathrm{sq}^{-1}$, and after immersion in the aniline polymerization solution for $1 \mathrm{~min}$, the sheet resistance increased to $167.0 \mathrm{M} \Omega \mathrm{sq}^{-1}$ due to the oxidation and etching of the $\mathrm{Cu}$ by APS. After immersion in the aniline polymerization solution for $3 \mathrm{~min}$, the film became nonconductive, as most of the Cu was etched away, and the amount of protonated PANI deposited in its place was not adequate to enable measurable conductivity. The conductivity of the PANI mesh then increased with increasing polymerization time up to $20 \mathrm{~min}$, at which point the sheet resistance reached the minimum value of $161.6 \mathrm{k} \Omega \mathrm{sq}^{-1}$. Then, the sheet resistance increased with increasing polymerization time above $20 \mathrm{~min}$, which could be attributed to the further oxidation of the synthesized PANI from the emeraldine form to the nonconductive pernigraniline form in the presence of the catalytic $\mathrm{Cu}$ ions. ${ }^{28}$ This transformation was confirmed by the change in 
(a)

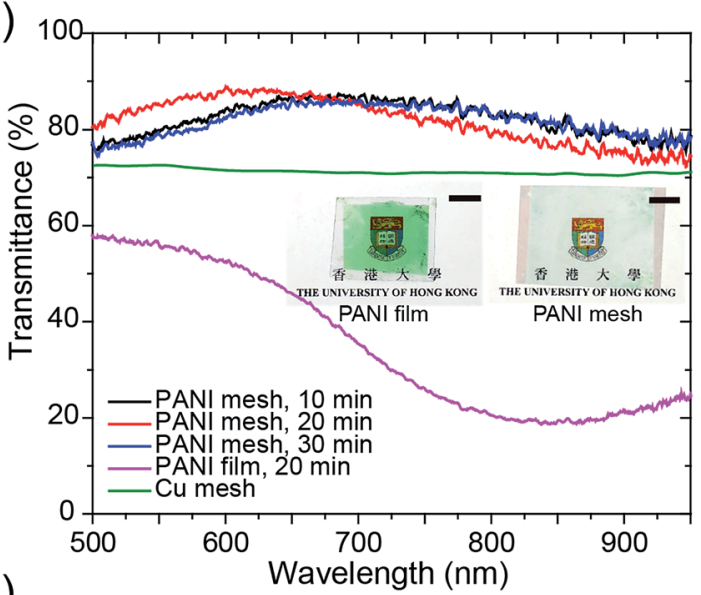

(b)

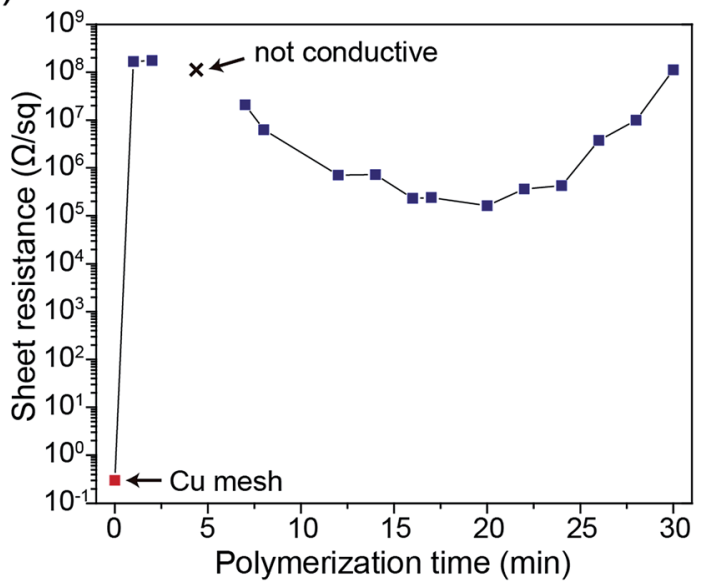

Fig. 3 Optical and electrical characterization. (a) Transmittance spectra of the initial $\mathrm{Cu}$ mesh template, PANI film and PANI meshes after $10 \mathrm{~min}, 20 \mathrm{~min}$, and $30 \mathrm{~min}$ in the aniline polymerization solution, respectively. (inset) Photographs of the PANI film (left) and the PANI micromesh (right) after $20 \mathrm{~min}$ in the aniline polymerization solution; the scale bars in the photograph represent $1 \mathrm{~cm}$. (b) Sheet resistance of the original $\mathrm{Cu}$ mesh and the PANI meshes after immersion in the aniline polymerization solution for various lengths of time ranging from $1 \mathrm{~min}$ to $30 \mathrm{~min}$

the appearance of the PANI film from green (emeraldine) to blue-green (mixture of emeraldine and pernigraniline), as displayed in Fig. S7 of the ESI. $\dagger$

To confirm the formation of a hierarchical nanostructured PANI mesh, the samples were characterized using Raman spectroscopy and X-ray diffraction (XRD). Fig. 4a presents the Raman spectra of the COC film, Cu mesh, PANI-coated COC film (PANI film) and PANI mesh. The aniline polymerization times for the PANI film and the PANI mesh were both $20 \mathrm{~min}$. For the bare COC film, the peak at $928 \mathrm{~cm}^{-1}$ was attributed to the norbornene units of COC, and the weaker peak at $1448 \mathrm{~cm}^{-1}$ was assigned to the $\mathrm{CH}_{2}$ bending mode. ${ }^{29}$ On the PANI film, only peaks at 1172 and $1333 \mathrm{~cm}^{-1}$ were observed, which can be attributed to in-plane $\mathrm{C}-\mathrm{H}$ bending and $\mathrm{C}-\mathrm{N}^{*+}$ stretching vibrations in PANI, respectively, because the signal of the underlying COC film was masked by the PANI film. The peaks observed at $928,1167,1319,1344$, and $1448 \mathrm{~cm}^{-1}$ in the Raman spectrum obtained from the PANI mesh reveal the presence of (a)
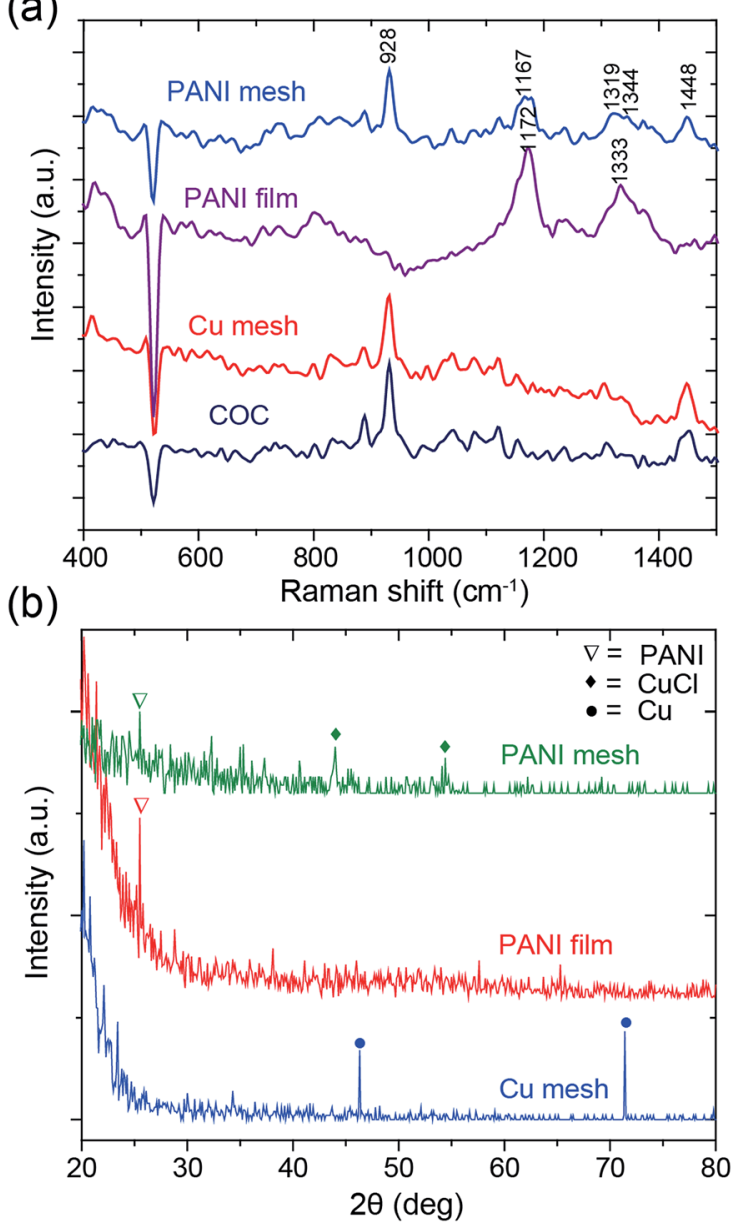

Fig. 4 (a) Raman and (b) XRD spectra of the COC film, Cu mesh, PANIcoated COC film (PANI film) and PANI mesh. The aniline polymerization times for the PANI film and mesh were both $20 \mathrm{~min}$.

PANI structures on the COC film. ${ }^{30}$ The shift in the peaks observed for the PANI mesh relative to the PANI film might be attributed to the interaction of PANI with $\mathrm{Cu}$ (II) chloride and its complex, which was produced by the oxidization of $\mathrm{Cu}$ by APS in the presence of hydrochloric acid.

The PANI polymerization process was further analyzed by XRD spectroscopy to determine the existence of etched $\mathrm{Cu}$. The spectral information of the samples is summarized in Fig. $4 \mathrm{~b}$. The peaks at $46.3^{\circ}$ and $71.4^{\circ}$ in the XRD spectrum of $\mathrm{Cu}$ mesh are attributed to the (111) and (220) planes of $\mathrm{Cu}$, respectively. ${ }^{31}$ For the PANI film and the PANI mesh samples, the characteristic peak of PANI was observed at $25.4^{\circ}$, which is attributed to crystalline PANI. The observation of peaks at $44^{\circ}$ and $54.4^{\circ}$, which are attributed to oxidized $\mathrm{Cu}$ (II) chloride (CuCl) and its complex, and the absence of $\mathrm{Cu}$ peaks in the spectrum of the PANI mesh confirmed the transformation. ${ }^{32}$ The oxidized $\mathrm{Cu}$ (II) also plays a key role in the catalytical deposition of the emeraldine form of PANI, as confirmed by a previous study. ${ }^{33}$ As displayed in Fig. S3 and $\mathrm{S} 5$ in the $\mathrm{ESI}, \uparrow \mathrm{Cu}(\mathrm{II})$ led to the formation of a dense and hierarchical nanostructured morphology of PANI.

The hierarchical nanostructured PANI mesh is a promising candidate for a high-performance ammonia gas sensor with 
high transparency and excellent flexibility. The sensing performance of the PANI mesh, PANI film, and $\mathrm{Cu}$ mesh for ammonia gas were experimentally investigated. The sensing sensitivity was defined as the normalized change in resistance, $S=\left(R_{\mathrm{g}}-R_{0}\right) / R_{0}$, where $R_{\mathrm{g}}$ is the resistance of the film after exposure to ammonia gas, and $R_{0}$ is the resistance of the film in air. Fig. 5a displays the real-time variation in the resistance of the $\mathrm{Cu}$ micromesh, hierarchical nanostructured PANI micromesh, and PANI film polymerized for 20 min upon exposure to different concentrations of ammonia gas ranging from $100 \mathrm{ppb}$ to $100 \mathrm{ppm}$. The response of the PANI mesh gradually increased with increasing concentration of ammonia and was higher than those of the PANI film, indicating that the small grain size increased the surface roughness of the hierarchical nanostructured PANI mesh. The increased roughness of the mesh resulted in a higher specific surface area, which led to a higher response upon exposure to the same concentration of ammonia as well as an enhanced signal-to-noise ratio (SNR) (Fig. S3 and S5, ESI $\dagger$ ). In contrast, the PANI film exhibited only half of the sensitivity to the same concentration of ammonia gas, which is attributed to the smoother surface on the PANI film, as well as the increased deposition of emeraldine form of PANI with the presence of catalytic $\mathrm{Cu}$ ions for the PANI mesh ${ }^{33}$ (Fig. S6, ESI $\dagger$ ). The detection limit of the PANI mesh for ammonia gas was as low as $2.5 \mathrm{ppb}$ (Fig. 5c), which makes the PANI mesh a superior candidate for the real-time, lowconcentration detection of ammonia gas. Fig. 5d demonstrates the variation in the sensing response as a function of the ammonia gas concentration; the curve implies that relationship between the response of our PANI mesh and the concentration of ammonia gas was highly linear (coefficient of determination $\left.r^{2}=0.988\right)$. Compared with other ammonia gas sensors constructed on hierarchical nanostructured PANI, our PANI sensor prototype showed an improved transparency with a comparable sensitivity. ${ }^{\mathbf{1 5 , 1 6 , 3 4}}$ Note that although a graphenepolyaniline nanocomposite film showed a slightly higher transparency $\left(92.5 \%\right.$ at a $550 \mathrm{~nm}$ wavelength) ${ }^{35}$ our PANI ammonia sensor prototype has a near-neutral color, a higher sensitivity (75 at $100 \mathrm{ppm}$ ammonia gas), and a simplified fabrication approach. (a)
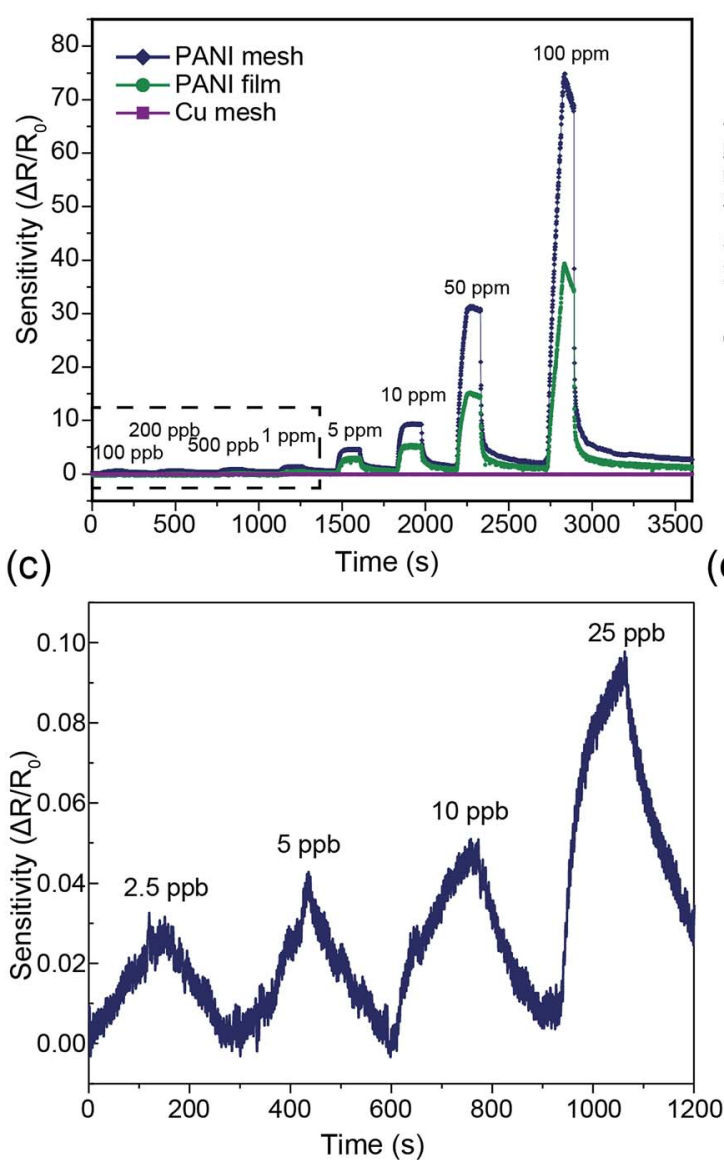

(b)
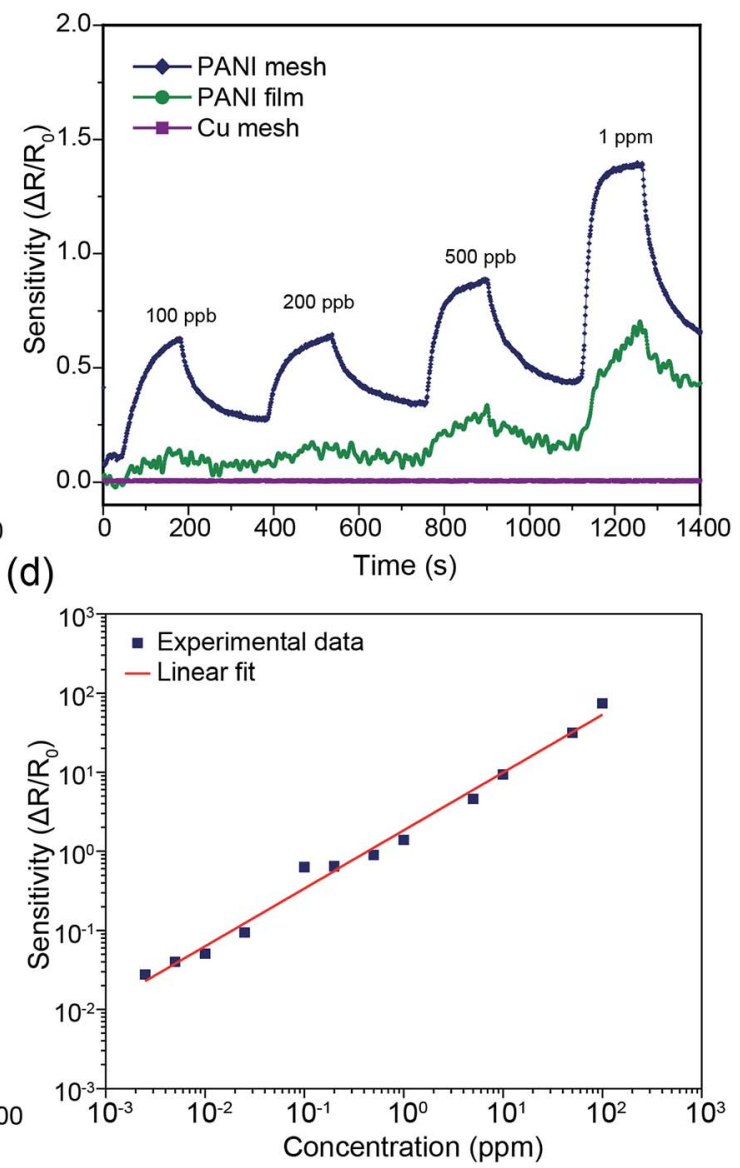

Fig. 5 (a) Sensing performance of the Cu mesh, PANI mesh and PANI film with different concentrations of ammonia gas ranging from 100 ppb to 100 ppm. The PANI mesh and PANI film were generated from 20 min aniline polymerization. (b) Enlarged plot showing the sensing performance of the Cu mesh, PANI film and PANI mesh with different concentrations of ammonia gas ranging from 100 ppb to 1000 ppb (the dashed box in (a)). (c) Sensing performance of the PANI mesh with different concentrations of ammonia gas ranging from $2.5 \mathrm{ppb}$ to $25 \mathrm{ppb}$. (d) Sensing sensitivity as a function of the ammonia gas concentration from $2.5 \mathrm{ppb}$ to $100 \mathrm{ppm}$. 
The selectivity among various gases is an essential parameter for any gas sensors. As displayed in Fig. 6a, the PANI mesh exhibited a specific response to ammonia gas over other volatile organic compounds. The gas sensing sensitivity of the PANI mesh to $10 \mathrm{ppm}$ ammonia gas was $136,165,497,79,137,262$, and 205 times higher than that to $10 \mathrm{ppm}$ ethanol, isopropanol, acetone, acetic acid, formaldehyde, toluene, and carbon dioxide, respectively. The PANI mesh also exhibited a slightly higher selectivity to ammonia than the PANI film did. Another important parameter of a gas sensor is the stability under interfering gases. Fig. $6 \mathrm{~b}$ shows the gas sensing stability of the PANI mesh to $20 \mathrm{ppb}$ ammonia gas with the presence of three other typically existing pollutant gases, formaldehyde, toluene, and carbon dioxide with range of $10 \mathrm{ppb}$ to $50 \mathrm{ppb}$, respectively. The variation of the sensitivity for all three gases were within $15 \%$, which further confirms the excellent selectivity and stability of PANI mesh ammonia sensor.
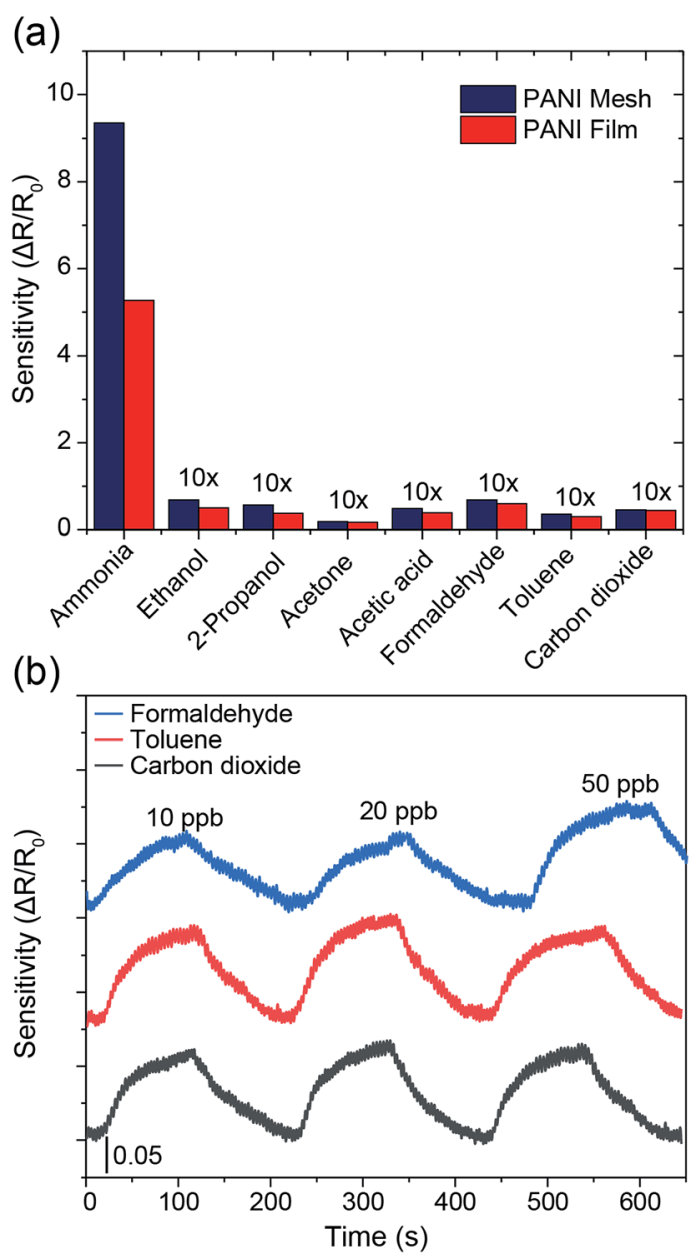

Fig. 6 (a) Gas sensing selectivity of the PANI mesh and PANI film for various volatile organic gases. The concentration of gas in all experiments was $10 \mathrm{ppm}$. The sensitivities for ethanol, 2-propanol, acetone, acetic acid, formaldehyde, toluene, and carbon dioxide are multiplied by 10 . (b) Ammonia gas sensing performance under different concentration of interfering gas species, including formaldehyde, toluene, and carbon dioxide ranging from $10 \mathrm{ppb}$ to $50 \mathrm{ppb}$. The concentration of ammonia gas was $20 \mathrm{ppb}$.
To examine the repeatability of the PANI mesh in highperformance ammonia gas sensing, the PANI mesh was exposed to $10 \mathrm{ppm}$ ammonia gas in four cycles, and no obvious decline in the sensing sensitivity was observed (Fig. S8, ESI†). The sensing performances of the PANI meshes generated from various polymerization times were also experimentally investigated, and the sample polymerized for 20 min exhibited the highest sensitivity (Fig. S9, ESI $\dagger$ ), which could be attributed to its higher proportion of emeraldine in the deposited PANI and the larger specific surface area than the other samples. For the sample produced with a shorter polymerization time, the deposited PANI was not adequate to form a complete mesh with full coverage in the trench. For the sample produced with a longer polymerization time, the overoxidation of the emeraldine form of PANI and the further growth of the PANI grains led to a decrease in the sensitivity of the mesh (Fig. S10, ESI†). The stability of the PANI mesh in high-performance ammonia gas sensing under various environment humidity was also examined by exposing to $20 \mathrm{ppb}$ ammonia gas, while the environmental relative humidity was maintained at $40 \%, 60 \%$, and $80 \%$, respectively, and the differences in the sensing sensitivity were less than $10 \%$ during the 15 min experiments, which is consistent with previous study ${ }^{36}$ (Fig. S11, ESI $\dagger$ ). However, when placed in the environment with high humidity for longer time, the sensitivity of the gas sensor may suffer further from the gradually increasing conductivity of the $\mathrm{HCl}$ doped PANI mesh. ${ }^{37}$

In addition to the enhanced sensing performance of the PANI mesh, the embedded nature of the PANI micromesh mitigated the risk of delamination from the substrate and enhanced its stability under mechanical bending. Fig. 7 provides the results of mechanical stability tests performed on the PANI ammonia gas sensor prototype under cyclic bending stress. The variation in the sheet resistance as a function of the number of cycles of tensile bending to a radius of $3 \mathrm{~mm}$ clearly

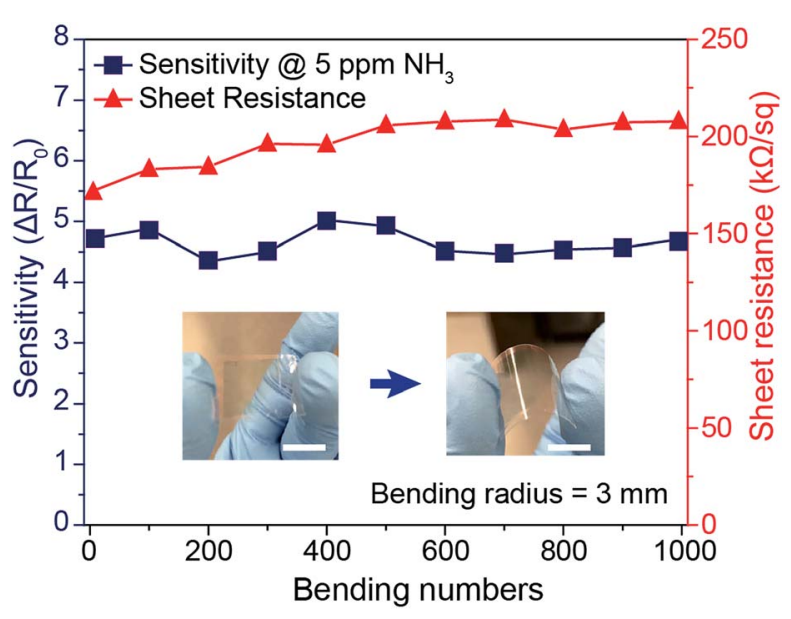

Fig. 7 Mechanical stability of the PANI ammonia gas sensor prototype. Variations in the sheet resistance (red curve) and sensing sensitivity (blue curve) to $5 \mathrm{ppm}$ ammonia gas versus the number of cycles of bending (tensile loading) to a radius of $3 \mathrm{~mm}$. The scale bars represent $1 \mathrm{~cm}$. 
indicates that the sheet resistance remained within $25 \%$ of its original value (in a range from $171.3 \mathrm{k} \Omega \mathrm{sq}^{-1}$ to $207.9 \mathrm{k} \Omega \mathrm{sq}^{-1}$ ). Meanwhile, the sensing sensitivity to $5 \mathrm{ppm}$ ammonia gas varied by only $14.4 \%$ over 1000 bending cycles. This remarkable stability of the PANI micromesh makes such devices promising candidates for use in flexible and wearable applications.

\section{Conclusion}

In summary, we have demonstrated a new type of highperformance electronic ammonia gas sensor with superior flexibility and excellent optical transmittance. The sensor was fabricated via in situ polymerization of aniline monomer on a catalytic sacrificial $\mathrm{Cu}$ micromesh template embedded in a flexible substrate prepared by solution-processed microfabrication. The hierarchical nanostructured PANI mesh increased the specific surface area relative to the PANI film, thus enhancing the sensitivity and SNR of the gas sensor. The assembled sensor exhibited high-performance sensing of ammonia gas with concentrations ranging from $2.5 \mathrm{ppb}$ to $100 \mathrm{ppm}$ and an excellent near-neutral color transparency (88.4\% at $600 \mathrm{~nm}$ wavelength). The embedded nature of the structure also improved the mechanical stability of the sensor under peeling and bending stress, and no obvious decrease in the sensing performance was observed after 1000 bending cycles. The synthetic approach can be easily adapted to prepare flexible and even stretchable sensors. With the superior performance and potentially low-cost, high-throughput fabrication process, our transparent PANI ammonia gas sensor holds the promise for broad applications in handheld or wearable flexible electronic or optoelectronic devices.

\section{Conflicts of interest}

There are no conflicts to declare.

\section{Acknowledgements}

This work was partially supported by the Research Grants Council of Hong Kong (Grant No. 27205515 and 17246116), the Department of Science and Technology of Zhejiang Province (Grant No. 2017C01058) and the University of Hong Kong (Grant No. 201611160057 and 201511159175$)$.

\section{Notes and references}

1 E. Westenbrink, R. P. Arasaradnam, N. O'Connell, C. Bailey, C. Nwokolo, K. D. Bardhan and J. A. Covington, Biosens. Bioelectron., 2015, 67, 733-738.

2 M. K. Choi, O. K. Park, C. Choi, S. T. Qiao, R. Ghaffari, J. Kim, D. J. Lee, M. Kim, W. Hyun, S. J. Kim, H. J. Hwang, S. H. Kwon, T. Hyeon, N. S. Lu and D. H. Kim, Adv. Healthcare Mater., 2016, 5, 80-87.

3 A. Romeo, T. S. Leung and S. Sanchez, Lab Chip, 2016, 16, 1957-1961.

4 Y. Park, B. Ryu, B. R. Oh, Y. J. Song, X. G. Liang and K. Kurabayashi, ACS Nano, 2017, 11, 5697-5705.
5 A. N. Abbas, G. Liu, B. L. Liu, L. Y. Zhang, H. Liu, D. Ohlberg, W. Wu and C. W. Zhou, ACS Nano, 2014, 8, 1538-1546.

6 X. T. Shuai, P. L. Zhu, W. J. Zeng, Y. G. Hu, X. W. Liang, Y. Zhang, R. Sun and C. P. Wong, ACS Appl. Mater. Interfaces, 2017, 9, 26314-26324.

7 S. Regonda, R. H. Tian, J. M. Gao, S. Greene, J. H. Ding and W. Hu, Biosens. Bioelectron., 2013, 45, 245-251.

8 Z. W. Liu, H. L. Wang and M. Cotlet, Chem. Mater., 2014, 26, 2900-2906.

9 R. E. de la Hoz, D. P. Schlueter and W. N. Rom, Am. J. Ind. Med., 1996, 29, 209-214.

10 J. F. Liu, X. Wang, Q. Peng and Y. D. Li, Adv. Mater., 2005, 17, 764-767.

11 K. K. Makhija, A. Ray, R. M. Patel, U. B. Trivedi and H. N. Kapse, Bull. Mater. Sci., 2005, 28, 9-17.

12 D. Bhattacharyya, K. Senecal, P. Marek, A. Senecal and K. K. Gleason, Adv. Funct. Mater., 2011, 21, 4328-4337.

13 Y. Yang, S. Li, W. Yang, W. Yuan, J. Xu and Y. Jiang, ACS Appl. Mater. Interfaces, 2014, 6, 13807-13814.

14 W. Huang, J. S. Yu, X. G. Yu and W. Shi, Org. Electron., 2013, 14, 3453-3459.

15 Y. X. Zhang, J. J. Kim, D. Chen, H. L. Tuller and G. C. Rutledge, Adv. Funct. Mater., 2014, 24, 4005-4014.

16 S. L. Bai, C. Z. Sun, P. B. Wan, C. Wang, R. X. Luo, Y. P. Li, J. F. Liu and X. M. Sun, Small, 2015, 11, 306-310.

17 Z. Q. Wu, X. D. Chen, S. B. Zhu, Z. W. Zhou, Y. Yao, W. Quan and B. Liu, Sens. Actuators, B, 2013, 178, 485-493.

18 M. Eising, C. E. Cava, R. V. Salvatierra, A. J. G. Zarbin and L. S. Roman, Sens. Actuators, B, 2017, 245, 25-33.

19 S. K. Mishra, S. N. Tripathi, V. Choudhary and B. D. Gupta, Sens. Actuators, B, 2014, 199, 190-200.

20 R. S. Andre, F. M. Shimizu, C. M. Miyazaki, A. Riul, D. Manzani, S. J. L. Ribeiro, O. N. Oliveira, L. H. C. Mattoso and D. S. Correa, Sens. Actuators, B, 2017, 238, 795-801.

21 J. F. Tian, G. Yang, D. G. Jiang, F. F. Su and Z. H. Zhang, Microchim. Acta, 2016, 183, 2871-2878.

22 N. Toshima, H. Yan and M. Ishiwatari, Bull. Chem. Soc. Jpn., 1994, 67, 1947-1953.

23 A. Puzari and J. B. Baruah, J. Org. Chem., 2000, 65, 23442349.

24 S. Virji, J. X. Huang, R. B. Kaner and B. H. Weiller, Nano Lett., 2004, 4, 491-496.

25 A. Khan, S. Lee, T. Jang, Z. Xiong, C. P. Zhang, J. Y. Tang, L. J. Guo and W. D. Li, Small, 2016, 12, 3021-3030.

26 A. Khan, S. Lee, T. Jang, Z. Xiong, C. P. Zhang, J. Y. Tang, L. J. Guo and W. D. Li, J. Visualized Exp., 2017, 124, e56019.

27 A. Khan, Y. T. Huang, T. Miyasaka, M. Ikegami, S. P. Feng and W. D. Li, ACS Appl. Mater. Interfaces, 2017, 9, 8083-8091.

28 W. J. Feast, J. Tsibouklis, K. L. Pouwer, L. Groenendaal and E. W. Meijer, Polymer, 1996, 37, 5017-5047.

29 J. Forsyth, J. M. Perena, R. Benavente, E. Perez, I. Tritto, L. Boggioni and H. H. Brintzinger, Macromol. Chem. Phys., 2001, 202, 614-620.

30 J. Xu, K. Wang, S. Z. Zu, B. H. Han and Z. Wei, ACS Nano, 2010, 4, 5019-5026. 
31 Y. Z. Zhang, G. F. Ding, P. Cheng and H. Wang, J. Electrochem. Soc., 2015, 162, D62-D67.

32 G. Marin, Z. Wang, G. Naterer and K. Gabriel, Int. J. Hydrogen Energy, 2011, 36, 13414-13424.

33 Z. Chen, C. Della Pina, E. Falletta and M. Rossi, J. Catal., 2009, 267, 93-96.
34 S. Chen and G. Sun, ACS Appl. Mater. Interfaces, 2013, 5, 6473-6477.

35 Y. L. Guo, T. Wang, F. H. Chen, X. M. Sun, X. F. Li, Z. Z. Yu, P. B. Wan and X. D. Chen, Nanoscale, 2016, 8, 12073-12080.

36 M. Matsuguchi, A. Okamoto and Y. Sakai, Sens. Actuators, B, 2003, 94, 46-52.

37 T. Taka, Synth. Met., 1993, 57, 5014-5019. 